\title{
Expressional Analysis of Inwardly Rectifying Kir4.1 Channels in Groggy Rats, a Rat Model of Absence Seizures
}

\author{
Yuya Harada ${ }^{1}$; Yuki Nagao ${ }^{1}$; Takahiro Mukai ${ }^{1}$; Saki Shimizu ${ }^{1}$; Kentaro Tokudome ${ }^{1}$; Naofumi \\ Kunisawa ${ }^{1}$; Tadao Serikawa ${ }^{1,2}$; Masashi Sasa ${ }^{3}$; Yukihiro Ohno ${ }^{1, "}$ \\ ${ }_{1}^{1}$ Laboratory of Pharmacology, Osaka University of Pharmaceutical Sciences, Takatsuki, Osaka, Japan \\ ${ }^{2}$ Institute of Laboratory Animals, Graduate School of Medicine, Kyoto University, Sakyo-ku, Kyoto, Japan \\ ${ }^{3}$ Nagisa Clinic, Hirakata, Osaka, Japan \\ ${ }^{*}$ Corresponding author: Yukihiro Ohno, Laboratory of Pharmacology, Osaka University of Pharmaceutical Sciences, Takatsuki, Osaka, Japan. Tel: +81-726901052, Fax: +81-726901053, \\ E-mail:yohno@gly.oups.ac.jp
}

Received: December 1, 2013; Revised: December 3, 2013; Accepted: December 5, 2013

\begin{abstract}
Background: The inwardly rectifying potassium channel subunit Kir4.1 is specifically expressed in astrocytes, which mediates spatial $\mathrm{K}^{+}$ buffering and is implicated in the pathogenesis of convulsive epileptic disorders (i.e. generalized tonic-clonic (GTC) and temporal lobe seizures).

Objectives: This study aimed to explore the pathophysiological role of Kir4.1 channels in modulating absence seizure incidence, using a spontaneously epileptic animal model.

Materials and Methods: Groggy rats, a rat model of human absence seizures, and Slc:Wistar (control)rats, were used in this study. Cortical and hippocampal EEG were recorded to confirm the seizure incidence in Groggy rats. The expression levels of Kir subunits (i.e. Kir4.1, Kir5.1 and Kir2.1) in ten brain regions were analyzed by Western blotting.

Results: Groggy rats showed a high incidence (ca. 350 seconds total duration/15 minutes observation period) of absence-like seizures, which were characterized by a sudden immobile posture and synchronously-associated spike and wave discharges. However, Western blot analysis revealed that Kir4.1 expression in Groggy rats was not significantly different from that of control rats in any of the brain regions examined (e.g. cerebral cortex, striatum, hippocampus, diencephalon, midbrain, pons/medulla oblongata and cerebellum). In addition, expressional levels of Kir5.1 and Kir2.1, which are also expressed in astrocytes, were unaltered in Groggy rats.

Conclusions: The present results suggest that unlike GTC and temporal lobe seizures, pathophysiological alterations (e.g. dysfunction and/or expressional changes) of Kir4.1 are not linked to non-convulsive absence seizures.
\end{abstract}

Keywords:Astrocytes; Rat; Epilepsy; Absence

\section{Background}

Inwardly rectifying potassium (Kir) channels are specifically expressed in astrocytes and mediate spatial $\mathrm{K}^{+}$ buffering, which removes excess extracellular $\mathrm{K}^{+}$at the synapses and maintains normal neuronal activities in the brain (1-7). These channels include; Kir4.1 channels, homo-tetramers of Kir4.1 subunits, and Kir4.1/5.1 channels, hetero-tetramers of Kir4.1 and Kir5.1 subunits, and conduct inward $\mathrm{K}^{+}$currents in response to a local elevation of extracellular $\mathrm{K}^{+}$concentration. In addition, spatial $\mathrm{K}^{+}$buffering is linked to glutamate uptake by astrocytes, which also influences neural excitability (8-12).

Recent clinical studies provide direct evidence that mutations in the human gene KCNJ10 encoding Kir4.1 evoke EAST (epilepsy, ataxia, sensorineural deafness, and tubulopathy) or SeSAME (seizures, sensorineural deafness, ataxia, mental retardation, and electrolyte imbalance) syndrome consisting of generalized tonic-clonic (GTC) seizures, ataxia, hearing loss and abnormal renal excretion of electrolytes $(13,14)$. All of the mutations in patients with EAST syndrome cause drastic decreases in $\mathrm{K}^{+}$ buffering currents mediated by both Kir4.1 and Kir4.1/5.1 channels, implying that the impaired functioning of Kir4.1 disrupts spatial $\mathrm{K}^{+}$buffering and causes epileptic seizures (15-17). In addition, it has also been shown that expressional levels of Kir4.1 were altered in animal models of epilepsy $(18,19)$, as well as in patients with temporal lobe epilepsy (20-22). All these findings strongly suggest that Kir4.1 channels are involved in the pathogenesis of GTC and/or temporal lobe seizures. However, there is no information as to whether Kir4.1 channels modulate nonconvulsive epileptic seizures, especially absence seizures. 
Harada Yet al.

\section{Objectives}

Therefore, in the present study, we performed Western blot analysis for Kir4.1 and Kir5.1 subunit expression using the Groggy rat, a newly developed animal model of absence seizures (23-25), to clarify the pathophysiological alterations of astrocytic Kir channels in absence seizures. The expressional level of the Kir2.1 subunit was evaluated since Kir2.1 channels are also known to be expressed in astrocytes even at low density $(26,27)$.

\section{Materials and Methods}

\subsection{Animals}

Groggy rats (GRY/Idr) of either sex at 17-19 weeks of age were obtained from the National BioResource Project for the Rat (NBRPR\#0368) in Japan. Age-matched male Slc:Wistar rats (SLC Japan, Shizuoka, Japan), the original strain of Groggy rats, were used as the control. Animals were housed in air-conditioned rooms under a 12 hour light/dark cycle (light on: 6:00 AM) and allowed ad libitum access to food and water. The housing conditions and the animal care methods complied with the guide for the care and use of laboratory animals of the Ministry of Education, Science, Sports and Culture of Japan. The experimental protocols of this study were approved by the Experimental Animal Research Committee at Osaka University of Pharmaceutical Sciences.

\subsection{EEG Recording}

Surgical procedures for the electrode implantation and EEG recordings were performed as described previously $(23,28)$. Groggy rats $(n=4)$ were anesthetized with pentobarbital (40 mg/kg, i.p.) and fixed in a stereotaxic instrument (Narishige SR-6, Tokyo, Japan). Small holes were made in the skull and silver ball electrodes were placed on the surface of the right or left frontal and occipital cortex. A reference electrode was placed on the surface of the frontal cranium. The electrodes were then connected to a miniature plug and fixed to the skull with dental cement. After a one week recovery period, animals underwent the subsequent EEG experiments. On the day of the EEG recording, the animals were placed in an electrically shielded observation cage $(28 \mathrm{~cm} \times 45 \mathrm{~cm} \times 20 \mathrm{~cm})$. After a 15 minutes habituation period, EEG and the behavior of the Groggy rats were simultaneously monitored for $15 \mathrm{~min}$ utes under freely-moving conditions, using an amplifier (MEG-6108; Nihon Kohden) and a thermal array recorder (RTA-1100; Nihon Kohden, Tokyo, Japan). Then the number of absence-like seizures (sudden immobile postures accompanying spike and wave discharges (SWD), and the total duration of SWD were calculated.

\subsection{Western Blot Analysis}

Groggy $(n=5)$, or control (Slc:Wistar, $n=6$ ) rats were deeply anesthetized with pentobarbital ( $80 \mathrm{mg} / \mathrm{kg}$, i.p.), and the brain was removed from the skull, then chilled in ice-cold saline. Next, the brain was dissected into the following 10 regions; frontal cortex (fCx), parieto-temporal cortex (ptCx), occipito-temporal cortex (otCx), striatum (St), hippocampus (Hpc), thalamus (Th), hypothalamus (Ht), midbrain (Mid), pons-medulla oblongata (P/MO) and cerebellum (Cer). The brain samples were homogenized in an ice-cold lysis buffer ( $\mathrm{pH}$ 7.5) containing: (in $\mathrm{mM}$ ) Tris 20, $\mathrm{NaCl} 150, \mathrm{MgCl}_{2}$ 10, EDTA 1.0, EGTA 1.0, $1 \%$ Triton X-100 and a mixture of protease inhibitors (leupeptin, aprotinin, E-64, pepstatin A, bestatin, and 4-(2-aminoethyl) benzenesulfonyl fluoride hydrochloride) (Nacalai Tesque, Kyoto, Japan). The homogenate was centrifuged at $15000 \mathrm{~g}, 4^{\circ} \mathrm{C}$ for 30 minutes and the supernatant was stored at $-80^{\circ} \mathrm{C}$ for the Western blot analysis.

Western blotting was performed according to the previously described methods $(18,19)$. Briefly, samples were incubated with a sodium dodecyl sulfate-polyacrylamide gel electrophoresis (SDS-PAGE) sample buffer for five minutes at $95^{\circ} \mathrm{C}$. Each sample $(20-40 \mu \mathrm{g} /$ lane $)$ was then subjected to SDS-PAGE and the separated proteins were transferred for 60 minutes to a PVDF membrane (GE Healthcare, Buckinghamshire, UK). The membrane was incubated first at room temperature for 60 minutes with a blocking solution containing 0.3-2\% skim milk in $25 \mathrm{mM}$ Tris, $150 \mathrm{mM} \mathrm{NaCl}, 0.1 \%$ Tween-20 (pH 7.5), then overnight $\left(4^{\circ} \mathrm{C}\right)$ with the corresponding primary antibodies, followed by a 60 minutes-incubation with a goat anti-rabbit IgG-HRP conjugate (1:2000, Santa Cruz Biotechnology, CA) for Kir4.1, donkey anti-goat IgG-HRP conjugate (1:2 000, Santa Cruz Biotechnology) for Kir5.1, a donkey antigoat IgG-HRP conjugate (1:2 000, Santa Cruz Biotechnology) for Kir2.1, or sheep anti-mouse IgG-HRP conjugate (1:2 000, GE Healthcare, Buckinghamshire, UK) for $\beta$-actin as the secondary antibody. Final detection was performed using the enhanced chemiluminescence method (Amersham ECL Western blotting detection reagents and analysis system, GE Healthcare, Buckinghamshire, UK) using a lumino imaging analyzer (LAS-3000, FUJIFILM, Tokyo, Japan). The primary antibodies used were a rabbit polyclonal antibody against Kir4.1 (1:500, Alomone Labs., Jerusalem, Israel), a goat polyclonal antibody against Kir5.1 (N-12) (1:400 Santa Cruz Biotechnology), a goat polyclonal antibody against Kir2.1 (1:400, Santa Cruz Biotechnology) and mouse monoclonal antibodies against $\beta$-actin (Sigma-Aldrich, St. Louis, MO). To normalize for protein loading, the chemiluminescence of the bands in each lane was standardized to the intensity of the $\beta$-actin band in the same lane.

\subsection{Statistical Analysis}

All data are expressed as mean \pm SEM. Expressional changes in Kir channel subunits determined by Western blot were compared with a Student's t-test (parametric two-group comparison). Differences were considered to be statistically significant for values of $\mathrm{P}<0.05$. 


\section{Results}

\subsection{Absence-like Seizures in Groggy Rats}

We first checked the frequency (incidence rate) of absence-like seizures in Groggy rats. Groggy rats showed ataxic gait with occasional extension of the hind limbs when moving, but other behaviors (eg, alertness, exploring and spontaneous activity) were generally normal. In addition, Groggy rats often exhibited spontaneous absence-like seizures, which are characterized by a sudden immobile posture with vacuous staring, accompanied by synchronously-associated EEG discharges characterized by $7-8 \mathrm{~Hz}$ SWD (Figure 1). The incidence of absence-like seizures and the total duration of SWD in the Groggy rats were $50.0 \pm 7.08$ times and $357 \pm 48.5$ seconds/15 minutes observation $(n=4)$, respectively.

Figure 1. Incidence of Absence-like Seizures and EEG Patterns in Groggy Rats

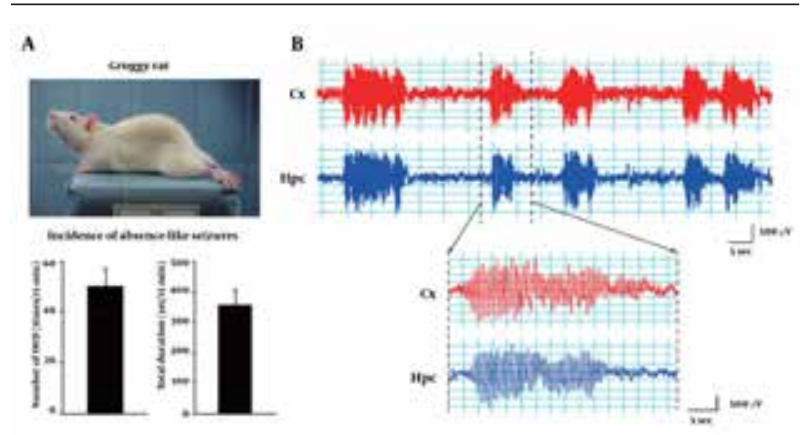

Groggy rats often exhibit spontaneous absence-like seizures with synchronously-associated 7-8 Hz spike-wave discharges (SWD). A) Number and total duration of absence-like seizures during a 15 minutes observation period. B) Typical EEG patterns recorded from the cerebral cortex (Cx) and hippocampus (Hpc) during absence-like seizures.

\subsection{Kir4.1 Expressional Levels in Groggy Rats}

As previously reported by both our group $(18,19)$ and other researchers $(29,30)$, Kir4.1 was detected primarily as a tetramer $(\sim 160 \mathrm{kDa})$ in all brain regions examined in Groggy and control (Slc:Wistar) rats (Figure 2). Levels of Kir4.1 were relatively high in the thalamus (Th), hypothalamus (Ht) and pons-medula oblongata $(\mathrm{P} / \mathrm{MO})$ in both animal groups (Figure 3). However, no significant differences in Kir4.1 expression were observed between Groggy rats and control rats in any of the 10 brain regions examined.
Figure 2. Western Blot Analysis for Kir4.1, Kir5.1 and Kir2.1 Subunits in Groggy Rats

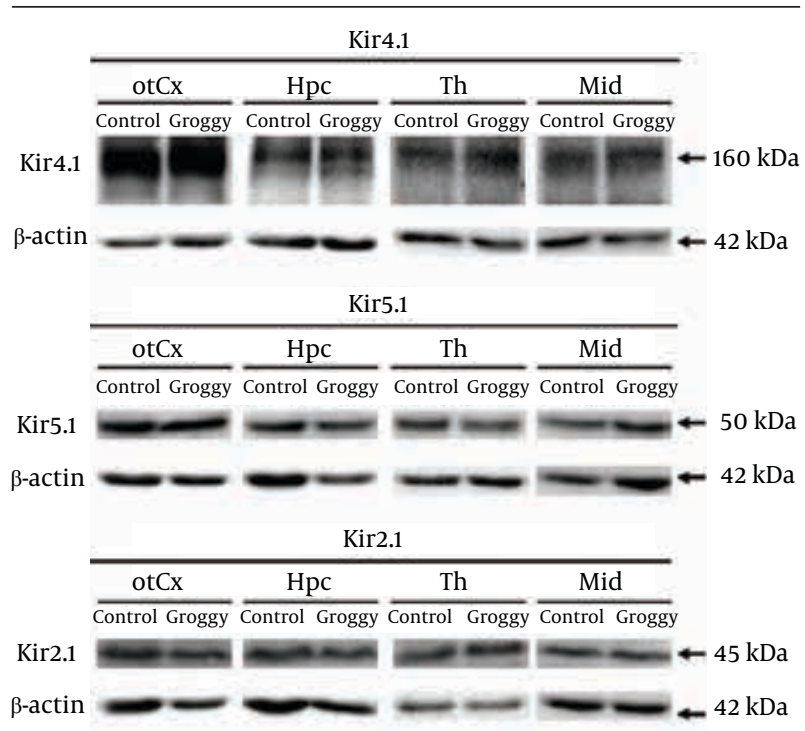

Figure 2 shows typical Western blots probed with the anti-Kir4.1, antiKir5.1 and anti-Kir2.1 antibodies in Groggy and control (Slc:Wistar) animals. otCx, occipito-temporal cortex; Hpc, hippocampus; Th, thalamus; Mid, Midbrain; Reference: $\beta$-actin.

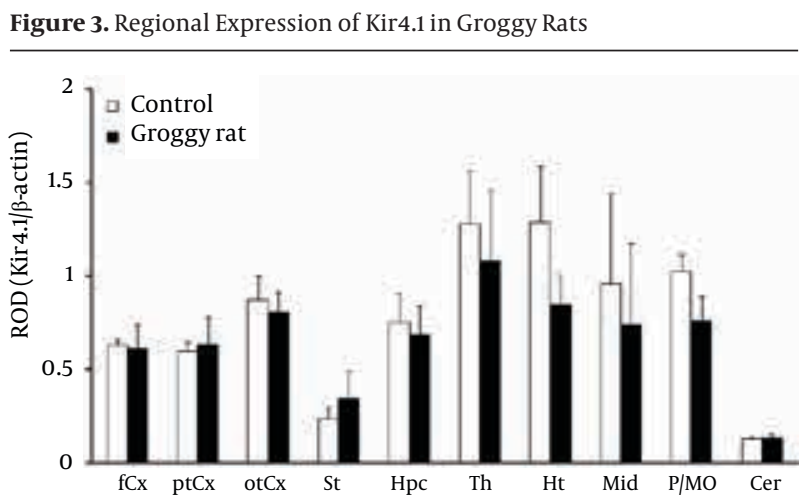

Kir4.1 expression was expressed as relative optical density (ROD) to $\beta$-actin. $\mathrm{fCx}$, frontal cortex; ptCx, parieto-temporal cortex; otCx, occipito-temporal cortex; St, striatum; Hpc, hippocampus; Th, thalamus; Ht, hypothalamus Mid, midbrain; P/MO, pons/medulla oblongata; Cer, cerebellum. Each value shows the mean \pm SEM of five (Groggy) or six (control) animals.

\subsection{Kir5.1 Expressional Levels in Groggy Rats}

As reported previously $(18,19)$, the Kir5.1 subunit was detected mainly as a monomer (Kir5.1:50 kDa) in all 10 regions (Figure 2). Levels of Kir5.1 were relatively high in the Th and midbrain (Mid) both in Groggy and control rats, but no statistical significances were observed between the two groups (Figure 4). 
Harada Y et al.

\subsection{Kir2.1 Expressional Levels in Groggy Rats}

The Kir2.1 subunit was detected as a monomer (Kir2.1: 45 $\mathrm{kDa}$ ) in all 10 regions (Figure 2). The levels of Kir2.1 were relatively high in the Th. However, the Kir2.1 levels in the 10 brain regions were not significantly different between the Groggy and control rats (Figure 5).

\section{Discussion}

Groggy rats, an ataxic mutant, were originally the progeny of female Slc:Wistar rats which had been given methylnitrosourea at an early stage of the gestational period (31). Groggy rats have thereafter been shown to carry a missense mutation (M251K) in the gene encoding the $\alpha_{1 \mathrm{~A}}$ subunit of the $\mathrm{P} / \mathrm{Q}$ type voltage-dependent $\mathrm{Ca}^{2+}$ channel (Cacna1a), and exhibit absence-like seizures (24). Specifically, Groggy rats frequently show a sudden immobile posture and synchronously-associated 7-8 Hz SWD in EEG. In addition, absence-like seizures in Groggy rats were selectively alleviated by medications for human absence seizures (i.e. ethosuximide and sodium valproate), but not by phenytoin, which lacks anti-absence activity (24). In the present study, we confirmed the incidence of absence-like seizures in Groggy rats, of which the frequency and duration for absence-like seizures were similar to those reported previously $(23,25)$. Since Groggy rats frequently exhibited absence-like seizures, expression analysis in the present study seems to reflect the Kir levels under an ictal state. However, neither expression of Kir4.1 nor Kir5.1 were changed in any brain regions of the Groggy rats, suggesting that Kir4.1 and Kir5.1 expression are not involved in epileptogenicity or consequent pathological changes in absence seizures. In addition, absence seizures also negligibly affected the expression of Kir2.1, which even at low levels is also expressed in the astrocytes of several brain regions (e.g. piriform cortex and olfactory bulb) $(26,27)$.

It is now known that dysfunction or reduced expression of Kir4.1 channels, due to gene mutations, induces GTC seizures and ataxia (e.g. unstable gait and/or frequent falls) in humans $(13,14)$. Although the detailed mechanisms remain to be clarified, we previously demonstrated that expression of astrocytic Kir4.1 was significantly reduced in Noda epileptic rats (NER), an epileptic model for GTC seizures, specifically in astrocytic foot processes in the amygdaloid nuclei (i.e. medial amygdaloid nucleus and basomedial amygdaloid nucleus) (18) (Table 1). In addition, recent clinical studies showed that Kir4.1 expression was significantly diminished in patients with temporal lobe epilepsy (20-22). All these results suggest that the reduced activity of astrocytic Kir4.1 channels evokes GTC and/or temporal lobe seizures, probably by disrupting spatial $\mathrm{K}^{+}$buffering, which consequently elevates extracellular $\mathrm{K}^{+}$and glutamate concentrations. Taken together, the present study revealed distinct roles of Kir4.1 in modulating convulsive and non-convulsive epileptic seizures and, unlike GTC seizures, the incidence of ab- sence seizures may not be affected by Kir4.1 or Kir4.1/5.1 channel activities and spatial $\mathrm{K}^{+}$buffering.

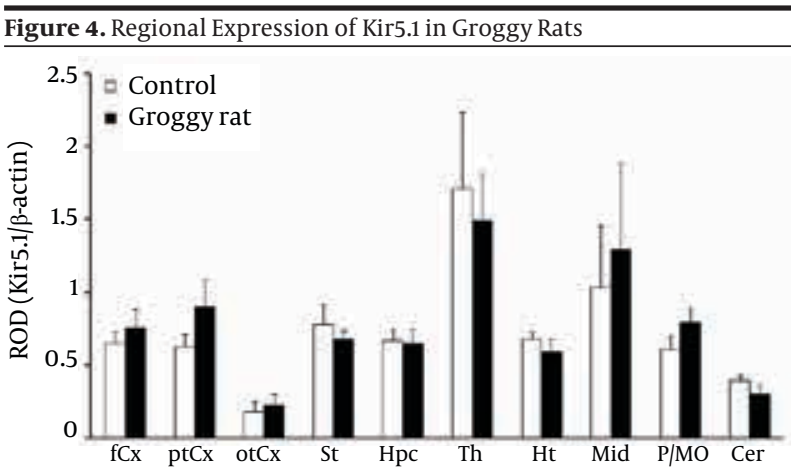

Kir5.1 expression was expressed as relative optical density(ROD) to $\beta$-actin. $\mathrm{fCx}$, frontal cortex; ptCx, parieto-temporal cortex; otCx, occipito-temporal cortex; St, striatum; Hpc, hippocampus; Th, thalamus; Ht, hypothalamus; Mid, midbrain; P/MO, pons/medulla oblongata; Cer, cerebellum. Each value shows the mean \pm SEM of five (Groggy) or six (control) animals.

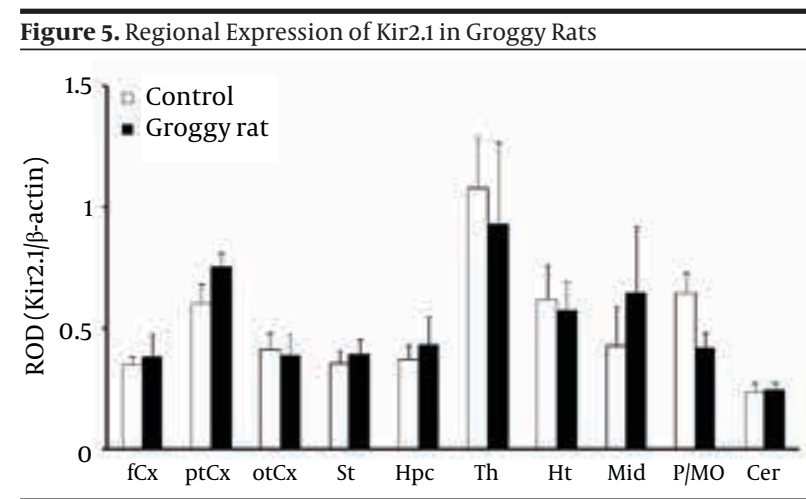

Kir2.1 expression was expressed as relative optical density(ROD) to $\beta$-actin. $\mathrm{fCx}$, frontal cortex; ptCx, parieto-temporal cortex; otCx, occipito-temporal cortex; St, striatum; Hpc, hippocampus; Th, thalamus; Ht, hypothalamus; Mid, midbrain; P/MO, pons/medulla oblongata; Cer, cerebellum. Each value shows the mean \pm SEM of five (Groggy) or six (control) animals.

Table 1. Alterations in Astrocytic Kir4.1 Expression in Anima Models of Absence-like and Generalized Tonic-clonic (GTC) Seizures ${ }^{\mathrm{a}, \mathrm{b}}$

\begin{tabular}{lll}
\hline Brain Regions & $\begin{array}{l}\text { GRY (Absence } \\
\text { Seizures) }\end{array}$ & $\begin{array}{l}\text { NER(GTC } \\
\text { Seizures) }\end{array}$ \\
\hline Frontal cortex & no change & no change \\
Parieto-temporal cortex & no change & no change \\
Occipito-temporal cortex & no change & $\downarrow$ c (amygdala) \\
Striatum & no change & no change \\
Hippocampus & no change & no change \\
Thalamus & no change & $\downarrow$ \\
Hypothalamus & no change & no change \\
Midbrain & no change & no change \\
Medulla-oblongata & no change & no change \\
Cerebellum & no change & no change \\
\hline
\end{tabular}

a Abbreviation: NER, Noda epileptic rats. GRY, Groggy rats

$\mathrm{b}$ The results for Kir4.1 expression in NER are quoted from our previous study (18).

C Reduced expression (down-regulation) 
In conclusion, to explore the pathophysiological alterations of Kir4.1 expression in absence seizures, Western blot analysis was performed in Groggy rats, a rat model of absence seizures. While Groggy rats showed a frequent incidence of absence-like seizures, our results revealed that neither the expression of Kir4.1, Kir5.1 nor Kir2.1 of Groggy rats was significantly altered in any of the brain regions examined (eg, cerebral cortex, striatum, hippocampus, diencephalon, midbrain, pons/medulla oblongata and cerebellum). The present results suggest that dysfunction of Kir4.1 is specifically linked to GTC and/or temporal lobe seizures, but not to absence seizures.

\section{Acknowledgements}

The authors would like to thank the National BioResource Project-Rat in Japan for providing the Groggy rats.

\section{Authors' Contribution}

Yuya Harada and Yuki Nagao contributed equally to this study. Design and leadership of group: Yukihiro Ohno; scientific consultation: Tadao Serikawa and Masashi Sasa; experiments, data collection and analysis: Yuya Harada, Yuki Nagao, Takahiro Mukai, Saki Shimizu, Kentaro Tokudome, Naofumi Kunisawa.

\section{Financial Disclosure}

The authors declare no financial disclosure.

\section{Funding/Support}

This work was partly supported by a Grant in Aid for Scientific Research (22590092) from the Ministry of Education, Science, Sports and Culture of Japan and a research grant from the Japan Epilepsy Research Foundation.

\section{References}

1. Butt AM, Kalsi A. Inwardly rectifying potassium channels (Kir) in central nervous system glia: a special role for Kir4.1 in glial functions. J Cell Mol Med. 2006;10(1):33-44.

2. Furutani K, Ohno Y, Inanobe A, Hibino H, Kurachi Y. Mutational and in silico analyses for antidepressant block of astroglial inward-rectifier Kir4.1 channel. Mol Pharmacol. 2009;75(6):1287-95.

3. Kofuji P, Newman EA. Potassium buffering in the central nervous system. Neuroscience. 2004;129(4):1045-56.

4. Ohno Y, Hibino H, Lossin C, Inanobe A, Kurachi Y. Inhibition of astroglial Kir4.1 channels by selective serotonin reuptake inhibitors. Brain Res. 2007;1178:44-51.

5. Simard M, Nedergaard M. The neurobiology of glia in the context of water and ion homeostasis. Neuroscience. 2004;129(4):877-96.

6. Su S, Ohno Y, Lossin C, Hibino H, Inanobe A, Kurachi Y. Inhibition of astroglial inwardly rectifying Kir4.1 channels by a tricyclic antidepressant, nortriptyline. J Pharmacol Exp Ther. 2007;320(2):573-80

7. Walz W. Role of astrocytes in the clearance of excess extracellular potassium. Neurochem Int. 2000;36(4-5):291-300.

8. Amiry-Moghaddam M, Ottersen OP. The molecular basis of water transport in the brain. Nat Rev Neurosci. 2003;4(12):991-1001.

9. Djukic B, Casper KB, Philpot BD, Chin LS, McCarthy KD. Conditional knock-out of Kir4.1 leads to glial membrane depolarization, inhibition of potassium and glutamate uptake, and enhanced short-term synaptic potentiation. J Neurosci. 2007;27(42):1135465.

10. Kucheryavykh YV, Kucheryavykh LY, Nichols CG, Maldonado HM Baksi K, Reichenbach A, et al. Downregulation of Kir4.1 inward rectifying potassium channel subunits by RNAi impairs potassium transfer and glutamate uptake by cultured cortical astrocytes. Glia. 2007;55(3):274-81.

11. Nagelhus EA, Horio Y, Inanobe A, Fujita A, Haug FM, Nielsen S, et al. Immunogold evidence suggests that coupling of $\mathrm{K}+$ siphoning and water transport in rat retinal Muller cells is mediated by a coenrichment of Kir4.1 and AQP4 in specific membrane domains. Glia.1999;26(1):47-54.

12. Puwarawuttipanit W, Bragg AD, Frydenlund DS, Mylonakou MN Nagelhus EA, Peters MF, et al. Differential effect of alpha-syntrophin knockout on aquaporin-4 and Kir4.1 expression in retinal macroglial cells in mice. Neuroscience. 2006;137(1):165-75.

13. Bockenhauer D, Feather S, Stanescu HC, Bandulik S, Zdebik AA, Reichold M, et al. Epilepsy, ataxia, sensorineural deafness, tubulopathy, and KCNJ10 mutations. N Engl J Med. 2009;360(19):196070.

14. Scholl UI, Choi M, Liu T, Ramaekers VT, Hausler MG, Grimmer J, et al. Seizures, sensorineural deafness, ataxia, mental retardation, and electrolyte imbalance (SeSAME syndrome) caused by mutations in KCNJ10. Proc Natl Acad Sci U S A. 2009;106(14):5842-7.

15. Reichold M, Zdebik AA, Lieberer E, Rapedius M, Schmidt K, Bandulik S, et al. KCNJ10 gene mutations causing EAST syndrome (epilepsy, ataxia, sensorineural deafness, and tubulopathy) disrupt channel function. Proc Natl Acad Sci US A. 2010;107(32):14490-5.

16. Sala-Rabanal M, Kucheryavykh LY, Skatchkov SN, Eaton MJ, Nichols CG. Molecular mechanisms of EAST/SeSAME syndrome mutations in Kir4.1(KCNJ10). J Biol Chem. 2010;285(46):36040-8.

17. Tang X, Hang D, Sand A, Kofuji P. Variable loss of Kir4.1 channel function in SeSAME syndrome mutations. Biochem Biophys Res Commun. 2010;399(4):537-41.

18. Harada Y, Nagao Y, Shimizu S, Serikawa T, Terada R, Fujimoto M, et al. Expressional analysis of inwardly rectifying Kir4.1 channels in Noda epileptic rat (NER). Brain Res. 2013;1517:141-9.

19. Nagao Y, Harada Y, Mukai T, Shimizu S, Okuda A, Fujimoto M, et al. Expressional analysis of the astrocytic Kir4.1 channel in a pilocarpine-induced temporal lobe epilepsy model. Front Cell Neurosci. 2013;7:104

20. Das A, Wallace GCt, Holmes C, McDowell ML, Smith JA, Marshall JD, et al. Hippocampal tissue of patients with refractory temporal lobe epilepsy is associated with astrocyte activation, inflammation, and altered expression of channels and receptors. Neuroscience. 2012;220:237-46.

21. Heuser K, Eid T, Lauritzen F, Thoren AE, Vindedal GF, Tauboll E, et al. Loss of perivascular Kir4.1 potassium channels in the sclerotic hippocampus of patients with mesial temporal lobe epilepsy. $J$ Neuropathol Exp Neurol. 2012;71(9):814-25.

22. Steinhauser C, Seifert G, Bedner P. Astrocyte dysfunction in temporal lobe epilepsy: $\mathrm{K}+$ channels and gap junction coupling. Glia. 2012;60(8):1192-202.

23. Ohno Y, Sofue N, Imaoku T, Morishita E, Kumafuji K, Sasa M, et al. Serotonergic modulation of absence-like seizures in groggy rats: a novel rat model of absence epilepsy. J Pharmacol Sci. 2010;114(1):99-105.

24. Tokuda S, Kuramoto T, Tanaka K, Kaneko S, Takeuchi IK, Sasa M, et al. The ataxic groggy rat has a missense mutation in the P/Q-type voltage-gated $\mathrm{Ca} 2+$ channel alpha1A subunit gene and exhibits absence seizures. Brain Res. 2007;1133(1):168-77.

25. Tokuda S, Sofue N, Ohno Y, Sasa M, Serikawa T. Inhibitory effects of levetiracetam on absence seizures in a novel absence-like epilepsy animal model, Groggy rat. Brain Res. 2010;1359:298-303.

26. Howe MW, Feig SL, Osting SM, Haberly LB. Cellular and subcellular localization of Kir2.1 subunits in neurons and glia in piriform cortex with implications for K+ spatial buffering. J Comp Neurol. 2008;506(5):877-93.

27. Kang SJ, Cho SH, Park K, Yi J, Yoo SJ, Shin KS. Expression of Kir2.1 channels in astrocytes under pathophysiological conditions. Mol Cells. 2008;25(1):124-30. 
28. Ohno Y, Ishihara S, Mashimo T, Sofue N, Shimizu S, Imaoku T, et al. Scn1a missense mutation causes limbic hyperexcitability and vulnerability to experimental febrile seizures. Neurobiol Dis. 2011;41(2):261-9.

29. Connors NC, Adams ME, Froehner SC, Kofuji P.The potassium channel Kir4.1 associates with the dystrophin-glycoprotein complex via alpha-syntrophin in glia. J Biol Chem. 2004;279(27):28387-92.
30. Seifert G, Huttmann K, Binder DK, Hartmann C, Wyczynski A Neusch C, et al. Analysis of astroglial K+ channel expression in the developing hippocampus reveals a predominant role of the Kir4.1 subunit. J Neurosci. 2009;29(23):7474-88.

31. Takeuchi IK, Takeuchi YK. Neuronal degeneration in the striatum of the groggy rat: a new mutant with a movement disorder. Experientia. 1991;47(11-12):1215-8. 